\title{
Reduction in Pediatric Growth Hormone Deficiency and Increase in Central Precocious Puberty Diagnoses During COVID 19 Pandemics
}

\section{Martina Peinkhofer}

University of Trieste: Universita degli Studi di Trieste

Benedetta Bossini ( $\sim$ benedetta.bossini27@gmail.com )

University of Trieste: Universita degli Studi di Trieste https://orcid.org/0000-0002-6046-1939

\section{Arturo Penco}

University of Trieste: Universita degli Studi di Trieste

\section{Manuela Giangreco}

Burlo Garofalo Pediatric Institute: IRCCS materno infantile Burlo Garofolo

\section{Maria Chiara Pellegrin}

Burlo Garofalo Pediatric Institute: IRCCS materno infantile Burlo Garofolo

\section{Viviana Vidonis}

Burlo Garofalo Pediatric Institute: IRCCS materno infantile Burlo Garofolo

\section{Giada Vittori}

Burlo Garofalo Pediatric Institute: IRCCS materno infantile Burlo Garofolo

Nicoletta Grassi

Burlo Garofalo Pediatric Institute: IRCCS materno infantile Burlo Garofolo

\section{Elena Faleschini}

Burlo Garofalo Pediatric Institute: IRCCS materno infantile Burlo Garofolo

\section{Egidio Barbi}

University of Trieste: Universita degli Studi di Trieste

\section{Gianluca Tornese}

IRCCS materno infantile Burlo Garofolo https://orcid.org/0000-0002-4395-3915

\section{Research}

Keywords: stimulation test, central precocious puberty, growth hormone deficiency, COVID-19

Posted Date: August 12th, 2021

DOI: https://doi.org/10.21203/rs.3.rs-734229/v1

License: (c) (i) This work is licensed under a Creative Commons Attribution 4.0 International License. Read Full License

Version of Record: A version of this preprint was published at Italian Journal of Pediatrics on March 28th, 2022. See the published version at https://doi.org/10.1186/s13052-022-01238-1. 


\section{Abstract}

Objective: The study aimed to evaluate differences in pediatric endocrine stimulation tests after the advent of COVID-19 pandemics.

Methods: Retrospective study with data collection for pediatric endocrine stimulation tests performed in 2019 e 2020 in a tertiary center.

Results: Overall, 251 tests were performed on 190 patients in 2020, compared to 278 tests on 206 patients in 2019 (-10\% tests; $-8 \%$ children evaluated). A significant reduction was found in tests to diagnose growth hormone deficiency (GHD) $(-35 \%)$, while LHRH tests increased (+22\%). A reduction of $30 \%$ in GHD diagnosis was observed. Diagnosis of central precocious puberty (CPP) increased by $38 \%$ compared to 2019 , mainly in females.

Conclusion: This study found a significant reduction of tests investigating GHD during COVID-19 pandemics. It also showed a clinically meaningful increase in cases of CPP in girls. These results suggest the need for families and pediatricians to monitor children's growth during isolation and enlighten new perspectives towards conditions associated with lockdown restrictions as increased screen time, social isolation, and children's anxiety as possible triggers of CPP.

\section{Introduction}

The two most frequent referral reasons in pediatric endocrinology are issues regarding growth and puberty, which constitutes almost half of the referrals for pediatric endocrinologists [1]. To detect growth hormone deficiency (GHD) or central precocious puberty (CPP), among other endocrine diseases, the determination of basal hormones is of limited diagnostic value, and stimulation tests - which require hospital admission - are needed.

Due to the COVID-19 pandemic, from March 9th, Italy was placed under the first national generalized lockdown: people across the entire peninsula, with unprecedented measures, were ordered to stay at home, and travels were banned, excluding only those for "urgent, verifiable work situations and emergencies or health reasons." To reduce pressure on hospitals and to minimize the hazard of direct person-to-person exposure, non-urgent visits were reduced. Also, schools of all levels, from kindergarten to universities, were kept closed for physical attendance, while teaching was pursued by telematic means. All activities gradually resumed from May 4th to June 15th, 2020, and until October 26th, when Italian authorities introduced new restrictions nationwide until May 2021.

While several studies have been published so far on the effect of COVID-19 pandemic on health care for non-COVID-19 diseases, mainly in pediatric emergency departments, indicating a reduction in both urgent and non-urgent visits [2-10], to date, no study evaluated the impact of the COVID-19 pandemic on the entire field of pediatric endocrinology.

This study aimed to evaluate differences between 2019 and 2020 in pediatric endocrine stimulation tests performed in a tertiary hospital.

\section{Material And Methods}

We conducted a retrospective study at the Institute for Maternal and Child Health IRCCS "Burlo Garofolo" in Trieste, Italy, a tertiary hospital and research institute that serves as a pediatric referral center for the province of Trieste and as a national reference hospital.

During COVID-19 pandemics, admissions for endocrine stimulation tests were among the procedures that were considered urgent and therefore not canceled. All records of children and adolescents performing a stimulation test from 
January 1st to December 31st, 2019 [11] and from January 1st to December 31st, 2020, were reviewed. The "G2 clinico" platform (management system specialist activities) was employed to access all patients' data. Information retrieved included age at presentation, gender, height SDS, BMI SDS, type and number of tests performed, reason(s) for referral, test results, and final diagnosis. Stimulation tests were performed and interpreted as previously described [11].

Ethical Committee approval was not requested since General Authorization to Process Personal Data for Scientific Research Purposes (Authorization no. 9/2014) declared that retrospective archive studies that use ID codes, preventing the data from being traced back directly to the data subject, do not need ethics approval [12]. Parents signed informed consent at the first visit, in which they agree that "clinical data may be used for clinical research purposes, epidemiology, the study of pathologies and training, to improve knowledge, care, and prevention."

Statistical analyses were mainly descriptive. Data are presented as frequencies and percentages or as median and interquartile ranges (IQRs) due to non-normal distribution. Fisher's exact test was used to evaluate associations between two categorical variables, while the non-parametric Wilcoxon Mann-Whitney test was applied to assess the difference in the distribution of a continuous variable across the two groups of a categorical variable. A P-value $<0.05$ was considered statistically significant. Analyses were performed with JMP ${ }^{\mathrm{TM}}$ software (version 16.1.0, SAS Institute Inc., Cary, NC, United States).

\section{Results}

Overall, while in 2019278 tests were performed on 251 patients in 2019 (47\% females) with a median age of 11.1 years (IQR 8.0;14.0), in 2020251 tests were performed on 190 patients ( $66 \%$ females, $p<0.05$ ) with a median age of 12.1 years (IQR 8.1;15.1); 59 individuals (29\%) in 2019 and 48 individuals (25\%) in 2020 performed more than 1 test.

The distribution of stimulation tests and pathological response is reported in Table 1. A significant difference among performed tests was found between 2019 and 2020 ( $p$ < 0.01) (Fig. 1): while in 2019 the most frequently performed test was ATT $(n=95,34 \%)$, in 2020 it was LHRHT $(n=81,33 \%)$. 
Table 1

Distribution of endocrine stimulation test according to suspected diagnosis and pathological tests in 2019 and 2020 (* ITT were performed to detect both GHD and CAI simultaneously)

\begin{tabular}{|c|c|c|c|c|c|c|c|}
\hline & & 2019 & & & 2020 & & \\
\hline $\begin{array}{l}\text { Suspected } \\
\text { diagnosis }\end{array}$ & $\begin{array}{l}\text { Stimulation } \\
\text { test }\end{array}$ & $\begin{array}{l}\mathrm{N} \text { of } \\
\text { performed } \\
\text { tests }\end{array}$ & $\begin{array}{l}\mathrm{N} \text { of } \\
\text { pathological } \\
\text { tests }\end{array}$ & $\begin{array}{l}\% \text { of } \\
\text { pathological } \\
\text { tests }\end{array}$ & $\begin{array}{l}\mathrm{N} \text { of } \\
\text { performed } \\
\text { tests }\end{array}$ & $\begin{array}{l}\mathrm{N} \text { of } \\
\text { pathological } \\
\text { tests }\end{array}$ & $\begin{array}{l}\% \text { of } \\
\text { pathological } \\
\text { tests }\end{array}$ \\
\hline \multirow[t]{4}{*}{ GHD } & ATT & 95 & 39 & $41 \%$ & 60 & 25 & $42 \%$ \\
\hline & $\begin{array}{l}\text { ATT + } \\
\text { GHRHT }\end{array}$ & 1 & 1 & $100 \%$ & & & \\
\hline & \multicolumn{4}{|l|}{ СTT } & 1 & 1 & $100 \%$ \\
\hline & ITT* & 31 & 25 & $81 \%$ & 19 & 16 & $84 \%$ \\
\hline $\mathrm{NC}-\mathrm{CAH}$ & SDST & 52 & 0 & $0 \%$ & 56 & 1 & $2 \%$ \\
\hline CPP & LHRHT & 48 & 24 & $50 \%$ & 54 & 28 & $52 \%$ \\
\hline \multirow[t]{3}{*}{ CAI } & ITT* & 31 & 5 & $16 \%$ & 19 & 4 & $21 \%$ \\
\hline & LDST & 16 & 1 & $6 \%$ & 13 & 1 & $8 \%$ \\
\hline & \multicolumn{4}{|l|}{ CRHT } & 1 & 0 & $0 \%$ \\
\hline $\mathrm{HH}$ & LHRHT & 18 & 5 & $28 \%$ & 27 & 1 & $4 \%$ \\
\hline AGHD & $\begin{array}{l}\text { ATT + } \\
\text { GHRHT }\end{array}$ & 17 & 0 & $0 \%$ & 15 & 1 & $7 \%$ \\
\hline $\mathrm{CH}$ & \multicolumn{4}{|l|}{ TRHT } & 1 & 1 & $100 \%$ \\
\hline OG & \multicolumn{4}{|l|}{ OGTT } & 4 & 2 & $50 \%$ \\
\hline \multicolumn{8}{|c|}{$\begin{array}{l}\text { (AGHD: Adult Growth Hormone Deficiency; ATT: Arginine Tolerance Test; ATT + GHRHT: Arginine Tolerance Test+ } \\
\text { Growth Hormone Releasing Hormone Test; CAl: Central Adrenal Insufficiency; CH: Central Hypothyroidism; CPP: } \\
\text { Central Precocious Puberty; CRHT: Corticotropin-Releasing Hormone Test; CTT: Clonidine Tolerance Test; GHD: } \\
\text { Growth Hormone Deficiency; HH: Hypogonadotropic Hypogonadism; ITT: Insulin Tolerance Test; LDST: Low-Dose } \\
\text { Synacthen Test; LHRHT: Luteinizing Hormone-Releasing Hormone Test; NC-CAH: Non-Classical Congenital Adrenal } \\
\text { Hyperplasia; OG: OverGrowth; OGTT: Oral Glucose Tolerance Test; SDST: Standard-Dose Synacthen Test; TRHT: } \\
\text { Thyrotropin Releasing Hormone). }\end{array}$} \\
\hline
\end{tabular}

GHD TESTS - Among tests evaluating a suspected GHD (arginine, insulin, and clonidine tolerance tests), a total reduction from 126 to 80 was detected (Table 1). GHD was diagnosed in 26 children in 2019 and 18 children in 2020 (Fig. 2). Median age at first stimulation test was 12.0 years (IQR 8.7;13.0) in 2019 and 12.5 years (IQR 9.0;13.5) in 2020 ( $p=$ 0.24). Overall, in 2020 there was a $40 \%$ reduction of patients evaluated for a suspected GHD with a decrease of $30 \%$ in diagnoses of GHD compared to 2019 , while the rate of positive tests was similar in the two years $(30 \%$ in $2019,35 \%$ in 2020). No differences were found in height SDS at first stimulation test between 2019 (median - 2.20 [IQR - 2.57;-1.79]) and 2020 (median - 2.17 [IQR - 2.71;-1.46]) ( $p=0.91)$.

LHRH TESTS - Details regarding LHRH tests are provided in Table 2. An increase of tests for a suspect of CPP in 2020 (+ $13 \%)$ was associated with a rise in pubertal responses $(+17 \%)$ compared to 2019 . Considering tests performed for suspected CPP, the number of diagnoses in females was higher in 2020 than 2019: 16 diagnoses in 2019 (42\% of tests) vs. 26 diagnoses in 2020 ( $58 \%$ of tests) $(p=0.03)$. 
Table 2

Details on LHRH tests performed in 2019 and 2020

\begin{tabular}{|c|c|c|c|c|c|c|c|}
\hline & & \multicolumn{3}{|l|}{2019} & \multicolumn{3}{|l|}{2020} \\
\hline & & Normal & Pathological & Total & Normal & Pathological & Total \\
\hline \multirow[t]{5}{*}{ Suspected diagnosis } & CPP & $24(50 \%)$ & $24(50 \%)$ & 48 & $26(49 \%)$ & $28(51 \%)$ & 54 \\
\hline & Females & 22 & 16 & 38 & 19 & 26 & 45 \\
\hline & Males & 2 & 8 & 10 & 7 & 2 & 9 \\
\hline & $\mathrm{HH}$ & $13(73 \%)$ & $5(27 \%)$ & 18 & $26(96 \%)$ & $1(4 \%)$ & 27 \\
\hline & Total & 66 & & & 81 & & \\
\hline
\end{tabular}

There was not a significant difference in BMI SDS for children with a suspect of CPP between 2019 (median 0.45 [IQR $0.16 ; 1.01]$ ) and 2020 (median 0.45 [IQR - 0.64;0.89]). While no significant differences were found in BMI SDS between all children diagnosed with CPP in 2019 (median 0.46 [IQR 0.13;1.01]) and in 2020 (median 0.17 [-0.30;0.74]), females diagnosed with CPP in 2020 had a BMI SDS (median 0.11 [IQR - 0.52;0.72]) significantly lower than those diagnosed in 2019 (median 0.93 [IQR 0.38;1.10]).

\section{Discussion}

In this retrospective study, we comprehensively analyzed differences in endocrine stimulation tests performed in a pediatric endocrinology outpatient department between 2019 and 2020 to detect any effect of COVID-19 pandemic restrictions.

Overall, we found a reduction of 10\% in tests and 8\% in children evaluated between 2019 and 2020, which was not related to hospital policies, since endocrine stimulation tests were considered urgent admissions during COVID-19 lockdown. The most significant detected changes were a reduction in tests to diagnose GHD (-30\% of patients diagnosed in 2020) and an increase in CPP diagnosis (+38\%, mainly females).

Regarding the tests investigating GH secretion, we detected a striking reduction (-35\%) in tests performed in 2020, compared to 2019. Although this is the first study reporting a decrease of referrals for suspected GHD during 2020 lockdowns, our results align with previous reports addressing a delay of hospital care admissions and a reduction of $30-40 \%$ in visits during the COVID-19 pandemic $[8,9]$. A possible explanation for the decrease in tests for suspected GHD is that well-child visits were canceled during the pandemics, removing a chance for family pediatricians to detect short stature and delayed growth. Moreover, families did not have the opportunity to compare their children with classmates (because of remote schooling and the absence of other social activities), missing possible deviations from the regular growth pattern to share with their family pediatricians. Although not considered in the present study, we have to contemplate that some families might have canceled their booked appointment because of the fear of going to the hospital during a pandemic or because they thought short stature was not an urgent issue. Since the rate of GHD among referred children was similar in the two years (30\% in 2019, 35\% in 2020) and coherent with previous data $[1,13]$ we probably missed 8 GHD diagnoses in 2020. Although these children might have been referred and eventually treated at a later stage, we know that the efficacy of treatment is increased if started at a younger age [14]. Moreover, approximately $35 \%$ of the patients with GHD have an organic etiology, and a delay in such a diagnosis can be life-threatening [15].

On the other side, our study found a clinically meaningful increase in CPP diagnoses in girls (+ 16\%) during 2020 compared to the same period in 2019. A "breast bud" appearance in a girl before the age of 8 years is always troublesome and easier to detect by families, even without comparisons with peers. Moreover, parents working from 
home might have increased the time spent with their children, detecting early pubertal changes more quickly. On the other hand, there was a decrease of $75 \%$ in CPP diagnosis in males. This reduction can be determined by the subtle finding of testicular enlargement (the earliest evidence of puberty in males) that patients and parents often go unnoticed

and can be missed if well-child visits from family pediatricians are missed. Our findings are in accordance with other two Italian reports: the first study found an increased incidence of newly diagnosed CPP in females with a faster rate of pubertal progression during COVID-19 pandemics compared to the same period, each year, from 2015 to 2019 [16]; similarly, in the second study, a rise in referrals for suspected precocious puberty was recorded in $2020(+108 \%$ consultations compared to 2019) [17]. These results seem to suggest a possible role of environmental factors on the early onset of puberty during COVID-19 pandemics. Precocious pubertal timing is crucial, resulting in a child sexually mature at an emotionally and socially inappropriate age, thus leading to risk-taking behaviors as sexual relations and substance use [18]. Genetics plays a significant role in CPP; however, environmental factors as obesity and adverse childhood experiences may influence pubertal development $[19,20]$. Since COVID-19 outbreak limitations forced children to home-schooling and sedentary lifestyle, a cross-sectional study found a change in dietary habits, with increased consumption of hypercaloric foods as pizza, bread, sweets, and more snacking, thus leading to overweight [21]. Considering these observations, we hypothesized a possible association of increased overweight secondary to COVID-19 restrictions and early puberty. Interestingly, we found no increase in BMI SDS between early pubertal girls observed in 2020. Our results align with an Italian report on referrals for precocious puberty in 2020, where no significant differences in anthropometric parameters were found compared to 2019 [17]. COVID-19 restrictions exposed children to e-learning and increased the use of screens. Stagi et al. found a more than doubled increase in time spent using electronic devices in children during the lockdown, hypothesizing its possible role in triggering puberty's early onset and tempo [16].

A potential limitation of this study is based on data collected from a single center; therefore, results may be related to the local population. On the other hand, to our knowledge, this is the first study that has simultaneously analyzed the features of pediatric endocrine stimulation tests as a whole during COVID-19 pandemics.

\section{Conclusion}

This study found a significant reduction of tests investigating GHD during COVID-19 pandemics, suggesting that a considerable number of children with GHD may have missed the opportunity of a timely diagnosis. It also showed a clinically meaningful increase in cases of CPP in girls. These results suggest the need for families and pediatricians to monitor children's growth during isolation and enlighten new perspectives towards conditions associated with lockdown restrictions as increased screen time, social isolation, and children's anxiety as possible triggers of CPP.

Since COVID-19 continues to represent a major global health threat challenging the accessibility to healthcare services, clinicians and the public health system should be aware that reduced access to health care is detrimental to both diagnosis and treatment of endocrine conditions.

\section{Abbreviations}

BMI SDS Body Mass Index Standard Deviation Score

CPP Central Precocious Puberty

GHD Growth Hormone Deficiency

IQR InterQuartile Ranges

\section{Declarations}




\section{Availability of data and materials}

The data that support the findings of this study are available from the corresponding author, $\mathrm{BB}$, upon reasonable request.

\section{Ethics approval and consent to participate}

Not needed.

\section{Consent for publication}

Not needed.

\section{Competing interests}

The authors have no conflicts of interest to report and no financial interests to disclose.

\section{Authors' contributions}

MP, AP, and BB conceptualized and designed the study, drafted the initial manuscript, designed the data collection instruments, collected data, and reviewed and revised the manuscript. MG carried out the statistical analysis and reviewed and revised the manuscript. VV, GV, and NG performed the stimulation tests and reviewed and revised the manuscript. EB conceptualized and designed the study and critically reviewed the manuscript for important intellectual content. GT conceptualized and designed the study, carried out the statistical analysis, drafted the initial manuscript, coordinated and supervised the data collection, and reviewed and revised the manuscript. All authors contributed to the article and approved the submitted version.

\section{Acknowledgements}

Not applicable.

\section{References}

1. Bellotto E, Monasta L, Pellegrin MC, et al. Pattern and features of pediatric endocrinology referrals: a retrospective study in a single tertiary center in Italy. Front Pediatr. 2020;8:580588.

2. Lazzerini M, Barbi E, Apicella A, et al. Delayed access or provision of care in Italy resulting from fear of COVID-19. Lancet Child Adolesc Health. 2020;4(5):e10-1.

3. Ciacchini B, Tonioli F, Marciano C, et al. Reluctance to seek pediatric care during the COVID-19 pandemic and the risks of delayed diagnosis. Ital J Pediatr. 2020;46:87.

4. Wise J. Covid-19: Delays in attending emergency departments may have contributed to deaths of nine children. BMJ. 2020;369:m2624.

5. Snapiri O, Rosenberg Danziger C, Krause I, Kravarusic D, Yulevich A, Balla U, et al. Delayed diagnosis of paediatric appendicitis during the COVID-19 pandemic. Acta Paediatr. 2020;109:1672-6.

6. Cozzi G, Zanchi C, Giangreco M, Rabach I, Calligaris L, Giorgi R, Conte M, Moressa V, Delise A, Poropat F. The impact of the COVID-19 lockdown in Italy on a paediatric emergency setting. Acta Paediatr. 2020;109(10):2157-9.

7. Rabbone I, Schiaffini R, Cherubini V, Maffeis C, Scaramuzza A. Diabetes Study Group of the Italian Society for Pediatric Endocrinology and Diabetes. Has COVID-19 Delayed the Diagnosis and Worsened the Presentation of Type 1 Diabetes in Children? Diabetes Care. 2020;43(11):2870-2. 
8. Ramos-Lacuey B, Herranz Aguirre M, Calderón Gallego C, et al. ECIEN-2020 study: the effect of COVID-19 on admissions for non-COVID-19 diseases. World J Pediatr. 2021;17(1):85-91.

9. Moynihan R, Sanders S, Michaleff ZA, et al. Impact of COVID-19 pandemic on utilisation of healthcare services: a systematic review. BMJ Open. 2021;11(3):e045343.

10. Raucci U, Musolino AM, Di Lallo D, et al. Impact of the COVID-19 pandemic on the Emergency Department of a tertiary children's hospital. Ital J Pediatr. 2021;47:21.

11. Penco A, Bossini B, Giangreco M, et al. Should Pediatric Endocrinologists Consider More Carefully When to Perform a Stimulation Test? Front Endocrinol (Lausanne). 2021;12:660692.

12. The Italian Data Protection Authority. Available at: https://www.garanteprivacy.it/web/guest/home/docweb/-/docweb-display/docweb/3786078 (Accessed July 2021). Authorisation no. 9/2014 - General Authorisation to Process Personal Data for Scientific Research Purposes.

13. Savage MO, Backeljauw PF, Calzada R, et al. Early detection, referral, investigation, and diagnosis of children with growth disorders. Horm Res Paediatr. 2016;85:325-32.

14. Polak M, Blair J, Kotnik P, et al. Early growth hormone treatment start in childhood growth hormone deficiency improves near-adult height: analysis from NordiNet® International Outcome Study. Eur J Endocrinol. 2017;177:4219.

15. Thomas M, Massa G, Craen M, et al. Prevalence and demographic features of childhood growth hormone deficiency in Belgium during the period 1986-2001. Eur J Endocrinol. 2004;151(1):67-72.

16. Stagi S, De Masi S, Bencini E, Losi S, Paci S, Parpagnoli M, Ricci F, Ciofi D, Azzari C. Increased incidence of precocious and accelerated puberty in females during and after the Italian lockdown for the coronavirus 2019 (COVID-19) pandemic. Ital J Pediatr. 2020;46(1):165.

17. Verzani M, Bizzarri C, Chioma L, et al. Impact of COVID-19 pandemic lockdown on early onset of puberty: experience of an Italian tertiary center. Ital J Pediatr. 2021;47(1):52.

18. Willemsen RH, Elleri D, Williams RM, Ong KK, Dunger DB. Pros and cons of GnRHa treatment for early puberty in girls. Nat Rev Endocrinol. 2014;10(6):352-63.

19. Reinehr T, Roth CL. Is there a causal relationship between obesity and puberty? Lancet Child Adolesc Health. 2019;3(1):44-54.

20. Zhang L, Zhang D, Sun Y. Adverse Childhood Experiences and Early Pubertal Timing Among Girls: A Meta-Analysis. Int J Environ Res Public Health. 2019;16(16):2887.

21. Sidor A, Rzymski P. Dietary Choices and Habits during COVID-19 Lockdown: Experience from Poland. Nutrients. 2020;12(6):165. 